\title{
Understanding the Importance of Time in Interior Architectural Design Method
}

\author{
Anak Agung Ayu Suci Warakanyaka ${ }^{1, *}$, Yandi Andri Yatmo ${ }^{1}$ \\ ${ }^{1}$ Department of Architecture, Universitas Indonesia, Indonesia
}

\begin{abstract}
The presence of time holds an important position in interior architectural practice and education. On theother hand, the presence of time challenges the stability and steadiness that framed the discipline. Furthermore, in interior architectural practice it is usually seen as a threat that should be either eliminatedor restricted. Rather than establishing defense mechanism against it, this paper argues that interiorarchitectural practice should be able to progress with time. By looking through undergraduate designstudio projects in Interior Architecture Program, Department of Architecture, Universitas Indonesia, this paper aims to addressed how the presence of time might affect, transform and even generate context specific interior architectural design methods that enables several dynamic forms of inhabitation. The out come of this study provides the opportunity for interior discipline to switch its focus, from the discipline that focuses on the aesthetic and constructional aspects, to the one that embraces the temporal aspectsof sociocultural conditions to enhance the wellbeing of its inhabitant
\end{abstract}

Keywords: time, transformative, interior architecture, design methods

\section{Introduction}

Architecture, and also the interior, has a quirky relationship with time. In both disciplines, time is seen more as a threat rather than as a benefactor. Time, with its ability to alter and transform space, is seen as the genesis of chaos and disorder, which is very much what both disciplines try to avoid. It becomes an intimidating terror that should be abolished or at least control in architecture and interior architectural practice. This dynamic quality of time is conflicting with the obsession of architecture and the interior in immortalising purity in space (Till, 2009). Especially with the discipline of the interior, which has long been responsible for controlling the physical forms of the space, is mostly threatened by the offensive presence of time that introduces the elements of change, unruly, and weariness. It has led the discipline of the interior to cast-out the influence of any temporal aspects and contingency in its practice and to developed a barricade mechanism to restrain it (Till, 2009).

Contrary to this argument, the interior architectural practice has no authority nor the ability to control time. Interior would never win the battle against time because the nature of the interior itself is ephemeral. Interior is a process of enabling inhabitation that always involves complicated spatial-temporal compositions. It is a transient process of becoming before it transforms into the rigid spatial entity (Attiwill, 2012). It is a being, rather than a setting, that presence largely depends on the way its inhabitant perceive space. The interior should be able to progress with time, not against it if it still wants to be able to accommodate the ever-changing needs of its inhabitant.

This paper aims to explore the importance of time in interior architectural practice and variety of design strategies it generates by looking through several undergraduate design studio projects in Interior Architecture Program, Department of Architecture, Universitas Indonesia. These projects addressed how the presence of time might affect, transform and even generate context-specific interior architectural design methods. It investigated the role of temporal elements, which are presented in the existing context, such as the layer of inhabitations, the everyday routine of the inhabitants and the sociocultural conditions in the context, that would act as the basis to produce timebased interior architectural design solutions.

The outcome of this exploration suggests that by acknowledging the presence of time, interior architecture could develop a ready in hand design tools that would subsequently produce responsive interior settings that would accommodate the changing needs of the inhabitants and also the environments. This study provides the opportunity for interior architecture discipline to widen the range of focus within its discipline, from the one that specifically concerns to the aesthetic and constructional aspects of the space, to the one that embraces the temporal aspects of sociocultural conditions in order to enhance the wellbeing of its inhabitant. This study could also contribute to enrich the theoretical tools in the interior architectural education

Corresponding author: ayuwarakanyaka@ui.ac.id 
that often suffered from its image as an intellectually lightweight discipline.

\section{The Power of Time}

Time, like the "outside", is a dreadful concept for the traditional interior architectural practice. Time, and so the "outside", is occupied by the immeasurable and the arbitrary (Bachelard, 2006), a concept in which the interior, the practice that was only operating from the "inside", could not fathom. It is the immediate threat to the value that the interior holds dear: control, order and familiarity. Time has the ability to produce temporal conditions such as weathering, programmatic changes, and accidents that would challenge the immutable authority of the interior architectural practice (Till, 1996). This correlation makes the practice of the interior develop certain attitude towards time. It either eliminates it all together or attempts to restrain any temporary possible variables in its design process.

Since modernity, the practice of the interior has been in a long battle to overpower time. It employs various strategies to help them reach a certain level of "purity", where they imagine that space is always in its perfect condition, regardless how harsh the reality is. It attempts to "freeze time, to hold onto that perfected moment of the completion of the building for as long as possible before and after the event" (Till, 1996, p.2). It utilises technology to banish any trace of time and generates the flawless, shiny and lasting image of the interior space where there is no dirt, accident and weather (Till, 1996). The architects and the designers believe that they have the full authority to set a certain limit to time to meet the terms of their design. This understanding has led the interior architectural practice to progress in the fairy tale, where it far removes from the contingent world which space will eventually occupy (Till, 2009). The interior believe that time is just an unproblematic universal, where they could get hold on to it on formal grounds (Till, 2009). It introduces time as aesthetic criteria, where it could be freeze and fold as a form or ornament. What they often do not realise is that this control is only achieved representationally and could never be applied in real life (Till, 1996). In reality, interior, as well as architecture, is a very dependent subject that affairs are not only determined by the architects as its "creator" but also contingent upon the spatial-temporal conditions of its inhabitant and its context.

The design of interior space is always vulnerable to the authority of the time. It exists IN time, not the other way around (Till, 1996). Acknowledging the importance of time does not mean that the interior practice should imitate the quality of time, let alone reproduce it, but to try to make sense of it "so as to know how to operate within them" (Till, 1996, p.5). By accepting that the interior is at the mercy of time, the practice of the interior should be conscious and progressing with the dynamic implications of time, such as programmatic and physical changes of the space. For this to happen, the interior architectural practice should shift its attention from the "superior cultural narratives", creating the perfect setting, to shift into the world of the everyday (Till, 1996). The everyday is the site of the ephemeral and it is by addressing the everyday, the interior architectural practice could generate an interior space that could accommodate the ever-changing needs of its inhabitant (Till, 1996).

\section{The Temporal Nature of Interior Practice}

The traditional practice of the interior, with it excessive fear of time, proposed that interior is a substantive being, which has a separate entity with the rest of its environment (Attiwill, 2012). But the recent theoretical progression of the discipline shown that the practice of the interior is shifting "from foregrounding things to processes, from the individual to process of individuation, from form to information, (and this is our highlight) from space to time and movement" (Attiwill, 2012 , p.1). Interior space is generated in the midst of flux and the product of the complex temporal-spatial compositions of its inhabitant and its context. It acknowledges that time has a crucial role in the production of the interior space in to enable inhabitation. The shift in the discipline is based on the thorough look on how our modern society inhabits space. The way we inhabit space changes from time to time according to the shift of sociopolitical affairs, technological advancement and the progression of lifestyle. At the moment, our society lives in the state of "atopia" where we are belonged nowhere except exactly in permanent "intransit-condition" (Verschaffel, 2012). This makes the interior, as the discipline that is supposed to provide inhabitation should be able to accommodate our nomadic ways of living. It should not be pre-determined for our mode of inhabitation that involves constant changes requires continuous adjustment.

By nature, the interior is dealing with the transformation of a given space; it is dealing with a situation that is continuously made and re-made (Brooker and Stone, 2007 and Attiwill, 2012). Interior space "'is not made just once, but is made and remade over and over again each time it is represented through another medium, each time its surrounding change, each time different people experience it" (Forty in Borden, 2006, p.50). This statement reflects that adjustment and temporality are the essence of the interior space in providing the physical and emotional wellbeing of its inhabitants. This adaptive nature of the interior is supported by a permeable boundary of the practice. Interior space is no longer just the room inside of the building; rather its territory is highly depending on how the body manoeuvre between the objects and space (Borden, 2006). It is not restricted to any physical form or placement; rather it is depending on its inhabitant state of affairs at the particular moment in time. Thus, interior should not be seen as a substantive being but as a process of becoming, as a product of a complicated relationship between space and time that enables inhabitation (Attiwill, 2012).

This made the interior architectural practice should develop a slightly different attitude towards time compared to the architectural practice. Dealing with an existing system makes the interior has to be a fleeting condition, where it supposed to be a set of "constantly shifting relationships between space and its users" (Lim, 2011, p.134). This is supported by McCarthy (2005) who 
stated that the practice of the interior is based on the interiority where it is not an absolute condition, rather it is a mobile and promiscuous situation, that generates the art of relationship where the limits is always redefined and renegotiated. It makes the interior not deal with the rigid layers of existing architectural space in what Frank Duffy called as "shearing layers of change" (in Brand, 1994). Instead, the interior is dealing with the most mundane, temporal and high-frequency layers of change such as the layer of service, space plan, stuff and soul (Brand, 1994). By addressing these layers of change in its design process, the interior architectural practice would generate an adaptive and responsive interior system that is able to progress with the dynamic needs of its inhabitants. This system adopts the system of nature where there is a continuous feedback loop of change that makes the inhabitation setting get to be harmonious (Brand, 1994). This understanding of time gives the possibility of the interior space to learn and progress together with its inhabitants.

There is a need to introduce interior architectural design method which includes tools, strategy and tactic that would focus on how to progressing along with the temporal aspect of inhabitation and also to cope with the unpredictability of the context. Moving away from the traditional conception of the interior, Attiwill (2013) suggests a proposition of "interiorizt" which makes the interior should not be seen as an object, entity or even space, but as a product of interiorization, "a making habitable through a process of selection and arrangement to produce an intensive space; a temporal consistency, a 'fabrication of space' which enables inhabitation" (Attiwill, 2012, p.5).

The idea of interiorizt suggests that the practice of the interior should not tackle the challenging nature of time, but instead should address it as "a creative problematic through design" (Attiwill, 2013, p.107). It makes the art of the interior is distinct from architectural tectonics where movement is not going to be frozen to generate certain ornamental form. But rather, movement, as one of the powerful components of time, becomes the main resources that reorient the way the interior practice organises, operates and dynamises its space (Attiwill, 2013). "An interior making through the production of a temporal consistency... becomes a composition of movement - "a rhythm" - that creates a space-time that enables inhabitation physically and mentally" (Attiwill, 2013, p.114).

Proposing a design method that is generated based on time and movement is a challenge to the conventional practice of the interior where the spatial programming and form finding is based on the acceptance of the inherited situations and its intolerable results. It opens up alternative ways of design thinking and address the interior as a discipline that depends on the dynamic nature of the physical and temporal situations of its environments. "In twenty-first-century design, a shift can be noted from a spatial emphasis to one of temporality in which the concept of the gesamtkunstwerk as a total work of art continues but become one of "temporary totalities, nomadic encampments or natural environments composed of aggregated assemblies"” (Attiwill, 2013, p.116). With this design proposition, it would be possible for the contemporary interior architectural practice to "achieves a sense of unity and cohesion that is provisional and dynamic, enabling engagement with contingency, temporality, and change" (Attiwill, 2013, p.116)

The key to creating the adaptive and responsive design is by putting certain levels of flexibility and allowing the design to be "wrong". Interior is the process that is made by displacements, that is why being "wrong" is the process of becoming. One of the tools to create the adaptive design is called the scenario planning, where it would not produce design plans but strategies (Brand, 1994, p.178). The differences are "where a plan is based on prediction, a strategy is designed to encompass unforeseeably changing conditions" (Brand, 1994, p.178). This creates rooms for possibilities where the inhabitants could manoeuvre according to their spatiotemporal needs, not the predefined interior. Although the regular spatial programming covers almost all the needs to create a responsive space, it is "leaving future users out of the picture, making the building all too optimal to the present and maladaptive for the future" (Brand, 1994, p.181). It adopts the law of ecology where "the more adapted an organism to present conditions, the less adaptable it can be to unknown future conditions" (Brand, 1994, p.181).

The scenario thinking allows architects and designers to start thinking the unthinkable (Brand, 1994). It develops "robust" strategy that would be viable in the variety of future (Brand, 1994). The scenario planning suggests a bottom-up approach that would let the interior space learns from its environment (Brand, 1994). By inviting and exploiting the diversity aspect of the everyday, the mundane and the ordinary scenario would emerge as a various combination of driving forces and organisation strategies that befitting the dynamic needs of its inhabitants (Brand, 1994). By allowing time to immerse in the design process, we are allowing for the emergence of the new modes of inhabitation. It also turns the occupants into an active collaborator of the interior space, where it would generate the feeling of intimacy, control and belonging.

\section{Interior Architectural Design Practice in Time}

The following chapter will explore the influence of time in generating design strategies in interior architectural design practice, by looking through two undergraduate design studio projects in Interior Architecture Program, Department of Architecture, Universitas Indonesia. All of these projects implemented the scenario planning with its bottom-up approach to address a particular design issue. These projects investigate the role of temporal elements in the existing context, such as the layers of inhabitations, the everyday routine of the inhabitants and the sociocultural context.

By exploring how these projects generate their design methods, we could investigate various adaptive and 
responsive interior architectural design strategies that would act as the tools to produce a time-based interior space that would accommodate the ever-changing needs of our contemporary society. The highlight of using the "robust" design strategies, as shown in these projects, is the ability of the interior space to respond to any immediate change that is initiated by its inhabitant and its environment. The scenario planning could prepare the interior space to cope with such changes.

\section{Transforming existing issue into design scenarios}

This project explores the issue of the waste distributions in Lokasari neighbourhood, Central Jakarta. This project started with investigating the mundane aspects of the neighbourhood, such as: the spatial programming of the street, the traffic flow, the habit and ritual of the actors, the atmosphere and the urban calendar.

This site is famous for its contrast atmosphere of daytime and nighttime. In daytime, the neighbourhood is mostly used as a site of formal commercial activities and parking lot. At night, the neighbourhood is famous for its entertainment and informal commercial activities, such as street vendors and street musicians. This project employed both behavioral and spatial mapping as a method to unfold the emerging issues on site. The mapping addresses all the variables that are responsible for the quality of the spatial experience in general.

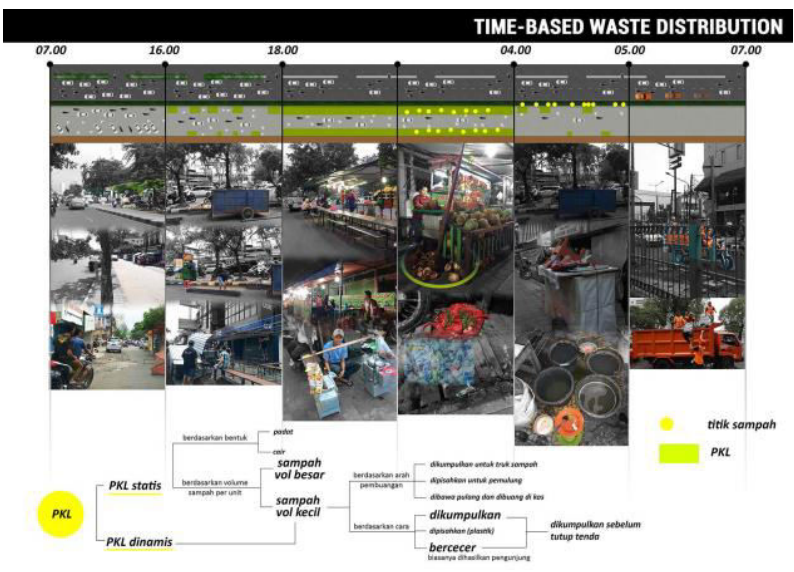

Fig. 1. Site Mapping.

Source: Kristofani, 2016.

One of the core issues was the distribution of liquid waste that affected all the activities in this neighbourhood. The production and distribution of the liquid waste involved all of the actors on site, such as: the street vendor, street musician, pedestrian, beggar and even the night escort. The mapping also found, because of the differences of activities in daytime and nighttime, some of the actors already employed the adaptive place making. For example, the street vendors use portable and collapsible tent/truck for their night commercial activities.

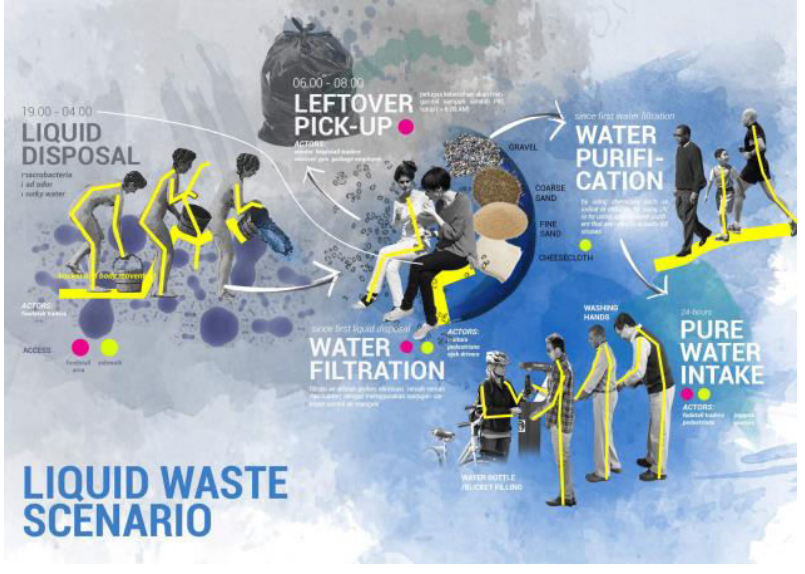

Fig. 2. Design Scenario

Source: Kristofani, 2016.

After thorough investigation, it is found that the issue could be transformed into design solution. All of the actors produced various types of liquid waste based on their consumption activities. The project attempts to exploit those consumption habit and tries to create an urban interior intervention that would not only solve the liquid waste issues, but also bring benefit and add value for the neighbourhood in general. It tries to cater multiple needs of the Lokasari's inhabitant at once.

Interior "deal with transformation of a given space" (Brooker and Stone, 2007, p.125). This argument is manifested in this project by trying to exploit the existing system to generate and support the new interior intervention. Utilizing the existing gutter and the water utility system, this project tried to address the issue of the liquid waste distribution by creating a water recycle system that could accommodate several activities surrounding water utilization. By creating scenario planning (Brand, 1994), this project came up with several possibilities on how this place would be used in both present time and also in the future (figure 2).

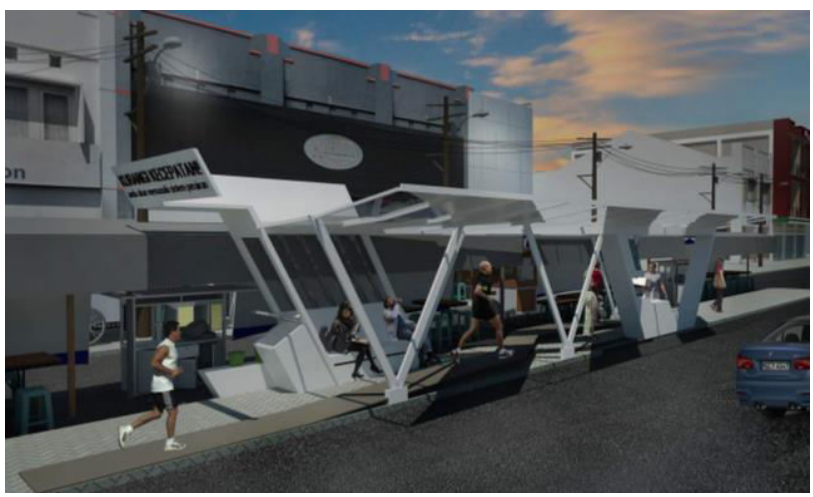

Fig. 3. Final Design.

Source: Kristofani, 2016.

The final design generate from this scenario planning was not substantially temporal or collapsible that could perform immediate response of physical changes. Yet, it is incorporating time in its design process that enables it to cope and adapt with the possible changes of the activities made by the inhabitants. It develops design solution with "robust" strategies to tackle on the heart of 
the problem and make use of technology, not to get rid of the waste or dirt, but to embrace it and transform it into design tools. This design strategy gives possibilities for the inhabitants to become an active dweller where they would keep the feedback loop and grow together with the interior intervention.

\section{Generating transformative urban interior setting}

Although located on the same site, this second project addresses a whole different issue from the first project: the issue of locality and cultural identity. Quite similar with the first project, it employed behavorial and spatial mapping in investigating the unseen sociocultural issues that might affected the quality of spatial experiences in the context. The mapping suggested that the context possessed an interesting history in the entertainment culture that was rooted far before the Indonesian independence.

Yet this locality was concealed by the current nightlife activities, which was only intended to and attracted particular target group and the presence of the street vendors, who contributed to crowdedness, waste, commodity exchange and lively atmosphere in the neighbourhoood. The site is currently developing an unpleasant image and there was almost nothing left from its former entertainment hub glory.
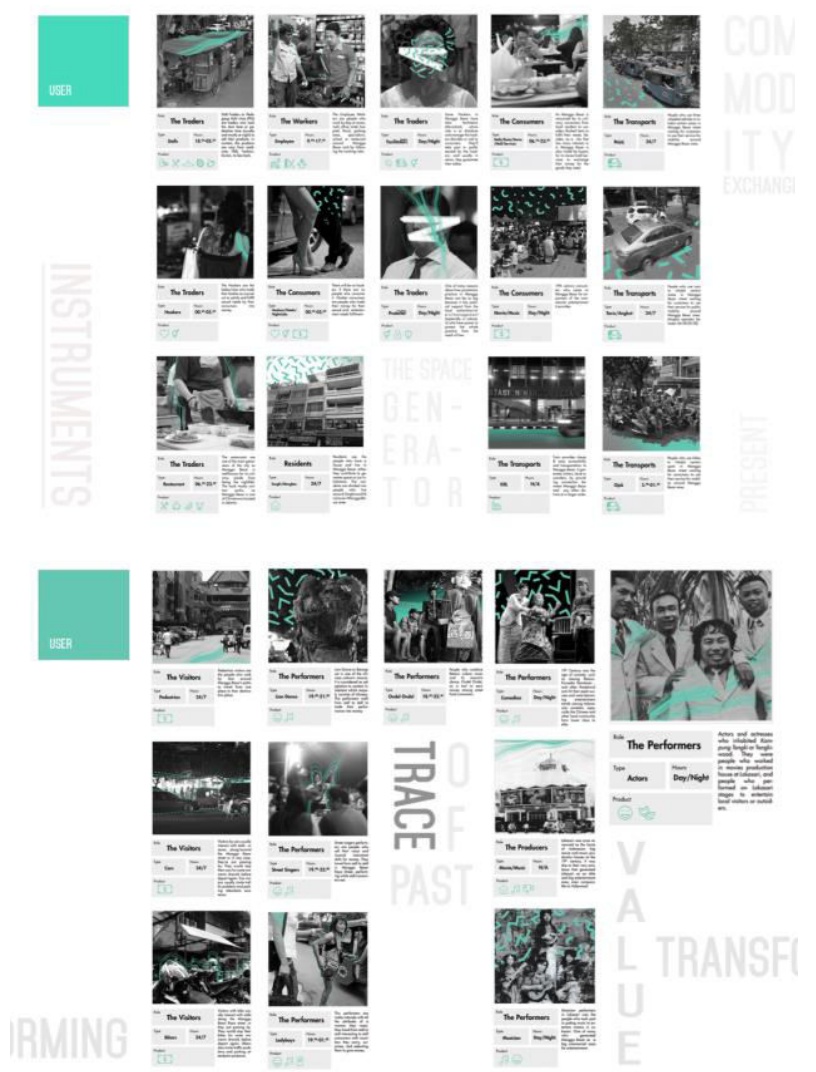

Fig. 4. User Behavorial Mapping.

Source: Kosasih, 2016.

The design solution generated from this issue was providing an urban interior setting that would accommodate all types of entertainment activities to claim back the image of Lokasari as Jakarta's entertainment hub without disturbing any existing commercial activities. By exploring the operation of how the street vendors organize and reorganize their stalls, this project proposes to create a transformative urban interior setting, therefore its forms, location and arrangement really depend on the demands of the inhabitants. In this project, the urban interior setting was generated through a series of modules and transmodules that would allow the interior setting to adapt with the immediate change of activities and accommodate various needs of entertainment activities.
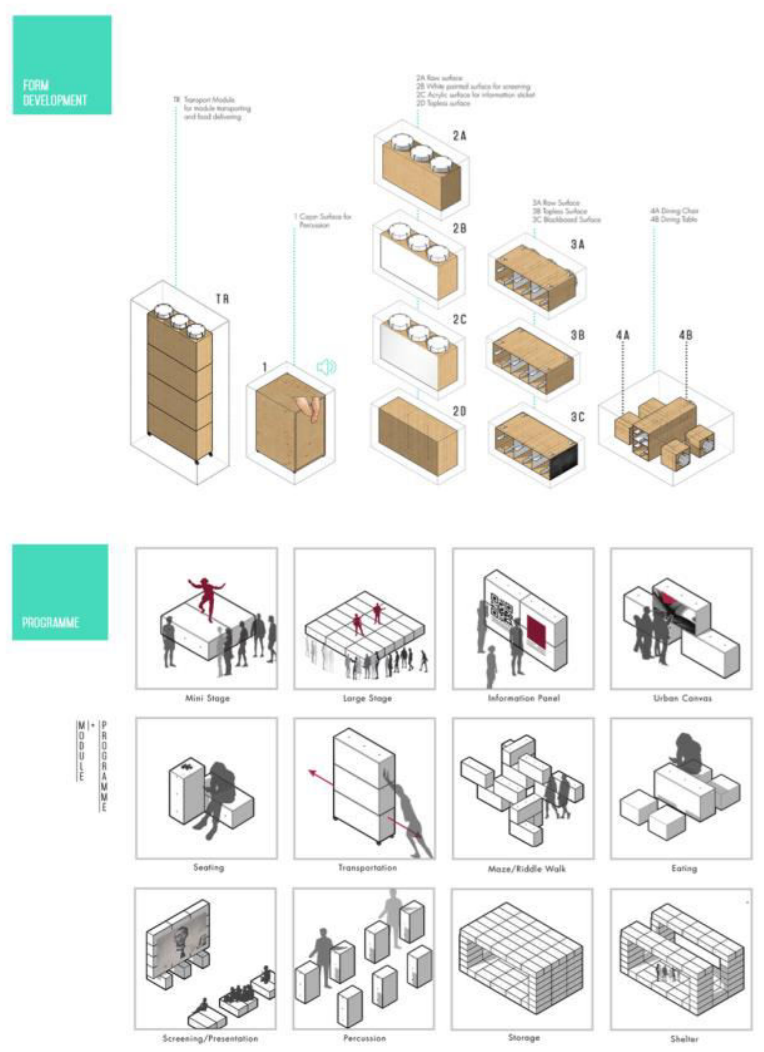

Fig.5. Design Scenario Diagram.

Source: Kosasih, 2016.

Aside from being able to generate various forms of temporary entertainment hub in any leftover spaces, these modules could be utilized to support some of the existing commercial activities in Lokasari. The details of scenario activities and transformative nature of the interior setting could be seen in Figure 5. Employing several features of collapsibility such as merging, adding, and retracting, these modules allow the inhabitants to freely adjust the arrangement of the modules to fit their spatiotemporal needs.

Rather than generating an exclusive interior setting to fulfill its purpose, this project brought the locality of the context - the entertainment culture, to the street where the activities actually happen. The interiorization of the street was made possible by employing the transformative spatial components that could adapt with the dynamic nature of the street. Rather than designing a rigid and high performative interior space, this project focused on exploring the ephemeral aspects of the 
interior to respond to the dynamic needs of the inhabitants.

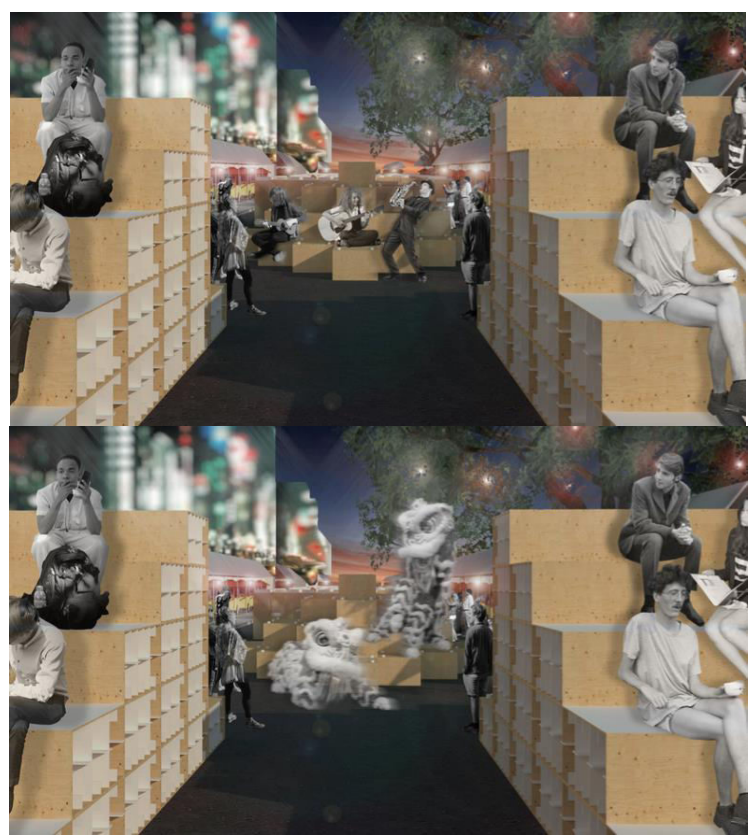

Fig. 6.Transformative Urban Interior Setting. Kosasih, 2016.

Both of these projects incorporates time on their design process and generate design strategies that is able to cope with the dynamic forces of the context. The first project use the bottom up approach to transform the existing issues into design solutions. It generates several design scenarios that would be able to catter multiple needs of the inhabitants. The second project use behavorial as well as spatial mapping to investigate the dynamic spatial operation that already existed in the context. The findings of this investigation is used to produce transformative urban interior setting that forms, location and arrangement responds the spatial-temporal situations of its inhabitants.

\section{Conlusions}

This paper argues that the presence of time is a crucial aspect in the progression of the interior architectural education. Rather than seeing time as intimidating forces, interior architecture should see it as the potential agency that would support the practice of interior architecture in enabling inhabitation. By incorporating time and its features in the design process, interior might be able to find suitable design tools to accommodate the ever-changing needs of the inhabitants. It might also generate context-specific interior architectural intervention, that not only addresses the current issues but is also well prepared for the future challenge of upcoming inhabitation. This exploration in design studio projects, illustrate that by employing scenario planning, interior architectural practice could generate adaptive and responsive spaces that could cope well with movement and changes. This study contributes to the enrichment of theoretical tools in the interior discipline that is aimed to provide inhabitation that enhances the physical and psychological wellbeing.

\section{Acknowledgements}

The projects and diagrams included in this paper are student works in Interior Architecture Design Studio 4 2017.

\section{References}

Attiwill, S. (2012). Beyond building: interior designs. IDEA Symposium 2012, (Interior: A State of Becoming), 1-7.

Attiwill, S. (2013). Interiorizt. In G. Brooker \& L. Weinthal (Eds.), The Handbook of Interior Architecture and Design (1st ed., pp. 107-116). London: Bloomsbury Academic.

Bachelard, G. (2006). The Dialectics of Outside and Inside. In M. Taylor \& J. Preston (Eds.), Intimus: Interior Design Theory Reader (pp. 22-25). Chichester: John Wiley \& Sons Ltd.

Borden, I. (2006). Thick Edge: Architectural Boundaries and Spatial Flows. In M. Taylor \& J. Preston (Eds.), Intimus: Interior Design Theory Reader (pp. 49-55). Chichester: John Wiley \& Sons Ltd.

Brand, S. (1994). How Building Learn: What Happen After They're Built. New York: Viking.

Brooker, G., \& Stone, S. (2007). From Organisation to Decoration. In Thinking Inside the Box: A Reader in Interior Design for the 21st Century. London: Middlesex University Press.

Lim, S. (2011). Tactics of The Peripatetic: On Vendors' Making an Interior of The Street. In Interior Tools Interior Tactics: Debates in Interiors Theory and Practice. Faringdon, Oxfordshire: Libri Pub.

McCarthy, C. (2005). Toward a Definition of Interiority. Space and Culture, Vol. 8 No. 2, 112-125

Till, J. (1996). Architecture in Space, Time. Architecture and Anthropology, (Academy Editions), 1-6.

Till, J. (2009). Architecture depends. Cambridge, Mass: MIT Press.

Verschaffel, B. (2012). The Meaning of Domesticity. In C. Briganti \& K. Mezei (Eds.), the Domestic Space Reader (p. 156). University of Toronto Press. 